\title{
Development and Validation of a New Measurement on Self-Contradiction
}

\author{
Yang $\mathrm{Wu}^{1}$ and Zhenzhen $\mathrm{Chen}^{2, *}$ \\ ${ }^{1}$ School of Marxism, Huazhong University of Science and Technology \\ ${ }^{2}$ Mental Health Center, Central China Normal University \\ *Corresponding author. Email: wuy3814@vip.qq.com
}

\begin{abstract}
Developing a clear, consistent, and positive self-concept of oneself is an important development task during early adulthood. Yet as people encounter innumerable shifts and changes in life, so should their self-concept. Studies show that inconsistencies in self-concept would appear constantly during adulthood, but currently, no suitable scale for direct measurement of the consistencies of self-concept exist. The present scale developed and validated the Self-Contradiction Scale, which consisted of three dimensions, using two separate samples with a total of 1852 participants. Overall, the psychometric properties of the new scale are satisfactory.
\end{abstract}

Keywords: Self-concept, Self-contradiction, Scale development, Psychometrics.

\section{INTRODUCTION}

Higgins (1987) identified three types of "incompatible self-beliefs" in the literature, which, though published more than three decades ago, are still relevant today: (a) the inconsistencies between external feedbacks and self beliefs held by a person, such as cognitive dissonance theory and self verification theory; (b) inconsistencies among various aspects of one's selfbeliefs, which would "impede a coherent and unified selfconcept", such as studies on dialectic self (Peng \& Nisbett, 1999; Spencer-Rodgers, Williams, \& Peng, 2010); and (c) inconsistencies between one's self-beliefs and certain standard, such as self-guide or social norm, such as self-discrepancy theory.

Surprisingly, as far as we know, three decades later there still have not been a comprehensive model on the extent of one's self-inconsistencies, accounting for its various aspects and providing a unified measurement tool.

In the present research, we define the contradiction of the self as one's perception about the inconsistency of his or her self-related information as well as its temporal instability. In this definition, the self-related information can be usefully subdivided according to its construal level or abstractness. Personality traits, self-beliefs, one's personal goals or ideals are at the conceptual level and are relatively abstract, whereas at the phenomenal level, less abstract self-related information may include a wide array of piecemeal information about the person, raw and unprocessed, such as a specific performance under a specific situation, evaluations from a specific judge and one's single behavior/thought/idea.

As such, the forms of inconsistency of self-related information could be threefold: a) contradictions among self-related information at the conceptual level; b) contradictions at phenomenal level; and c) contradictions across the conceptual and phenomenal levels, for example, a person who believes himself to be a highachieving student but finds out his exam score to be low (akin to "discrepancies between one's self-perceived attributes and some standard or self-guide"; Higgins, 1987). The second part of the self-contradiction concept involves the stability of self-related information. The higher the expectation of self as about to change dramatically in the future, the less stable one's perception of one's self would be, implying a higher possibility of having an internally inconsistent self (Chandler \& Lalonde, 1998; Nurra \& Oyserman, 2018).

The aim of this study is to develop and validate a measure of individual's contradictions within perceived self-related information, thus paving the way for further investigation. The scale construction was mostly theoretically driven and was consistent with the definition given earlier. 


\section{METHOD}

\subsection{Participants}

Two independent samples from several universities in China were recruited. Sample 1 has 912 valid participants (487 men, 425 women, 2 unreported; $M_{\text {age }}=20.000$, $S D=1.380$ ), and Sample 2 has 261 valid participants (50 men, 204 women, 7 unreported; $M_{\text {age }}=19.323$, $S D=1.090)$. Of the two sample, Sample 1 is groupadministered and as a result are relatively more susceptible to response bias including careless responding, therefore we added an item to identify and exclude carelessly responded cases from the analysis; Sample 2 was collected in a laboratory setting with ample time for participants and were presumed less affected by these biases.

Another two undergraduate samples were independently recruited for testing test-retest reliability. Sample 3 has 51 valid participants (19 men, 31 women; $\left.M_{\text {age }}=22.098, S D=11.159\right)$ recruited during the spring semester of 2016, and Sample 4 has 628 valid participants (414 men, 209 women, 5 unreported; $M_{\text {age }}$ $=17.960, S D=.685)$ recruited during the fall semester of 2017. Participants in both Sample 3 and 4 were measured twice, and the time interval between two waves are 2 weeks for Sample 3 and 16 weeks for Sample 4.

\subsection{Measures}

\subsubsection{Self-contradiction Scale}

According to the theoretical framework aforementioned, the construct of self-contradiction could be subdivided into three dimensions: contradictions within one's self-concept (Internal Contradictions), the contradictions between the concept and feedbacks (Feedback Contradictions) and the perception of the temporal changes (Self-Change). An initial pool of 20 items were generated by interviews, theoretical analysis and by revising items from published scales assessing similar constructs. Nearly half of the items are negatively worded to control for acquiescence bias, and we changed background grey of certain keywords in some items to aid in the word segmentation of Chinese words. An independent sample of 197 participants pilot-tested the initial scale, and a focus group consisted of three nonpsychology major students discussed the wording of items. As a result, we deleted three items due to content ambiguity that are open to misunderstanding. The final version used in the study consisted of 17 items, of which 8 items are negatively worded, with 5 items measuring Internal Contradiction and 6 items for Feedback Contradiction and Self Change respectively. Responses were anchored on a 7-point Likert scale.

\subsubsection{Self-esteem}

Rosenberg's (1965) self-esteem scale was utilized to measure participants perception of self-worth. The scale consists of 10 items, half of which are negatively worded. The scale adopts a 7-point Likert scale in this study, ranging from 1 "strongly disagree" to 7 "strongly agree". Cronbach's alpha of the RSES is .806 .

\subsubsection{Self-concept Clarity}

The Chinese version of Campbell et al. (1996)'s selfconcept clarity scale was employed to measure the clarity of participants' self-concept. Participants rate 12 items (e.g. "In general, I have a clear sense of who I am and what I am"), of which 9 items are negatively worded (e.g. "Sometimes I think I know other people better than I know myself") on a 7-point Likert scale (ranging from 1 "strongly disagree" to 7 "strongly agree"). Cronbach $\alpha$ in current research is .739.

\subsubsection{Big Five Measures}

We measured the Big Five personality factors with the revised Chinese version of the Big Five Inventory (John, Donahue, \& Kentle, 1991), which consists of 42 items that were rated on a 5-point Likert scale.

\subsubsection{Dialectical Self}

We also included the Dialectical Self Scale developed by Spencer-Rodgers, Boucher, Mori, Wang, and Peng (2009) to provide validity evidence for our selfcontradiction scale. The DSS scale consists of 32 items, with 15 negatively worded items and measures respondents' general inclination for a dialectical thinking style. Cronbach $\alpha=.739$.

\subsubsection{Other Measure.}

Based on Meade and Craig's (2012) suggestions, we included one item ("I have been to all the country in the world") placed in the middle of the survey to identify careless responded cases. Cases in which participants rated this item other than 1 ("Strongly disagree") were considered problematic and deleted list-wise.

\section{ANALYSIS AND RESULTS}

\subsection{Reliability and Exploratory Factor Analysis}

The overall reliability of the scale in two samples, measured in Cronbach's alpha, is .766 and .754, respectively. The Cronbach's alpha ranged from .618 to .753 among three subscales of the two samples, with an exception of lowest value of .570 in self-change subscale in Sample 1. 
Sample 3 and Sample 4 were used to document the test-retest reliability of the Self-Contradiction Scale across different time span. The cross-temporal stability was estimated using correlational analyses of participants' responses at Time 1 and Time 2. In Sample 3 , the overall test-retest correlation is .787 , and ranges from .801 to .610 for the three dimensions. In Sample 4 where data are collected during a much longer time span, the overall test-retest correlation is .503, and ranges from .604 to .462 for the three dimensions.

To test the factorial structure of self-contradiction scale, we used Sample 1 to derive a preliminary version of the scale by exploratory factor analysis (EFA) and confirmatory factor analysis (CFA), and Sample 2 was used to cross-validate this factorial structure using CFA. The EFA on Sample 1 extracted common factors using principal axis factoring with varimax rotation, and the number of factors were determined by the "eigenvalue 1 " threshold and scree plot. Using the approach, results revealed a 4-factor solution in which the loadings of one item, Item 13, were below .400 on all factors. An inspection of the factors suggested that the item loadings generally fitted our hypothesized model, and the additional factor may stem from an artifact of item wording (Podsakoff, MacKenzie, \& Podsakoff, 2012) since all the negatively worded items loaded onto one factor, or the "method factor".

\subsection{Confirmatory Factor Analysis.}

A more rigorous test on the method factor and the suitability of Item 13 was conducted using confirmatory factor analysis. Under the multitrait-multimethod framework, a series of "correlated trait, correlated methods" models (CTCM models), in which additional one or two factors were specified to items sharing the same wording method (Weijters, Baumgartner, \& Schillewaert, 2013), were used to test the presence of method artifacts occasioned by item wording (Lindwall et al., 2012). To avoid overfitting, we fitted the models to both samples and compared the results. The three critical questions addressed in CFA were: a) the viability of the theoretical three-factor model $b$ ) the existence of method bias c) the need to remove Item 13.

Based on the three questions, we specified 8 models to be tested. Model 1 specified a unidimensional model with all 17 items loaded onto one latent factor. Model 2 included a substantive factor as in Model 1 and specified factors for both positively and negatively worded items. Model 3 is an extension of Model 2, with Item 13 deleted. Model 4 is the hypothesized three-factor model. Based on Model 4, Model 5 and Model 6 both specified an additional method factor for negatively worded and positively worded items, respectively. Model 7 included factors for both wording methods. Model 8 is similar to Model 7, only with Item 13 deleted.

We grouped the 8 models into 2 blocks. The first block consisted of Model 1-3, the comparison among which may help determine the existence of method bias and the deletion of Item 13 in a unidimensional model. The second block consisted of Model 4-8, which variants of CTCM models are compared to pinpoint the source of wording effect and also help determine the status of Item 13. The comparison between unidimensional and threefactor-model is evident from comparing models between the two blocks. Our hypothesis is that the three-factor model with Item 13 deleted and with two method factors would best fit the data.

Table 1. Fit Indices of self contradiction scale (Sample 1)

\begin{tabular}{|l|l|l|l|l|l|l|l|l|}
\hline Model & $\chi^{2}$ & df & RMSEA & SRMR & AIC & NNFI & CFI & GFI \\
\hline 1 & 1884.445 & 119 & .145 & .112 & 2484.586 & .676 & .716 & .764 \\
\hline 2 & 627.490 & 101 & .079 & .049 & 787.581 & .886 & .915 & .920 \\
\hline 3 & 555.581 & 87 & .080 & .050 & 698.558 & .888 & .918 & .924 \\
\hline 4 & 1460.330 & 116 & .129 & .121 & 1968.063 & .747 & .784 & .805 \\
\hline 5 & 711.675 & 108 & .080 & .088 & 838.986 & .878 & .903 & .912 \\
\hline 6 & 863.906 & 107 & .092 & .086 & 1034.087 & .845 & .878 & .892 \\
\hline 7 & 413.443 & 98 & .060 & .054 & 534.751 & .930 & .949 & .948 \\
\hline 8 & 281.631 & 84 & .052 & .049 & 393.879 & .951 & .966 & .962 \\
\hline
\end{tabular}


Table 2. Fit Indices of Self Contradiction Scale (Sample 2)

\begin{tabular}{|c|c|c|c|c|c|c|c|c|}
\hline Model & $\chi^{2}$ & $\mathrm{df}$ & RMSEA & SRMR & AIC & NNFI & CFI & GFI \\
\hline 1 & 657.650 & 119 & .139 & .114 & 786.768 & .578 & .631 & .755 \\
\hline 2 & 283.985 & 101 & .086 & .066 & 410.785 & .831 & .875 & .878 \\
\hline 3 & 242.120 & 87 & .085 & .065 & 349.336 & .843 & .887 & .892 \\
\hline 4 & 409.678 & 116 & .107 & .099 & 536.512 & .764 & .799 & .827 \\
\hline 5 & 268.726 & 108 & .074 & .067 & 353.160 & .861 & .890 & .894 \\
\hline 6 & 294.779 & 107 & .083 & .081 & 391.238 & .837 & .871 & .881 \\
\hline 7 & 148.921 & 98 & .042 & .056 & 253.831 & .952 & .965 & .939 \\
\hline 8 & 123.245 & 84 & .040 & .048 & 223.009 & .959 & .971 & 946 \\
\hline
\end{tabular}

Table 1 and 2 each provided a summary of fit indices of these models. The comparison of these models confirmed existence of method bias by showing that models with explicit method factors generally fitted data better than those without (i.e. Model 1 and Model 4, respectively). Moreover, compared with Model 4, the CTCM models (Model 5-7) generally fitted data better, and the goodness-of-fit indices is highest when both method factors of positively and negatively worded items were specified (Model 7). Lastly, the comparison of Model 7 and Model 8 revealed that removal of Item 13 could significantly increase the fit ( $\mathrm{ps}<.02)$. The result pattern is comparable across the two samples, further lending support to our hypothesis. Overall, the comparison of models supported our hypothesis of the three-factor-model.

\subsection{Correlations with Related Measures}

We calculated the factor scores (DiStefano, Zhu, \& Mîndrilă, 2009; Grice, 2001) of the three factors according to Model 8 using sem package (Fox, 2006; Fox, Nie, \& Byrnes, 2015) in R (R Core Team, 2015), and calculated the zero-order correlations between the factor scores of self-contradiction and other related constructs (Table 3).

Results revealed moderate, positive correlations between two factors of self-contradiction (internal and feedback contradiction) and dialectic self ( $r=.362$ and .387) and a negative correlation with self-concept clarity $(r=-.509$ and -.560). These provided initial evidence for the convergent validity of self-contradiction. Furthermore, internal and feedback contradiction negatively correlated with self-esteem, openness, extraversion, conscientiousness and agreeableness, while positively correlated with neuroticism. The self-change dimension, on the other hand, showed significant correlations with these psychological variables in an opposite direction.

Table 3. Correlation between Self-contradiction scales and other constructs

\begin{tabular}{|l|l|l|l|l|l|l|l|l|l|l|l|}
\hline & 1 & 2 & 3 & 4 & 5 & 6 & 7 & 8 & 9 & 10 & 11 \\
\hline 1.Internal contradiction & 1.000 & & & & & & & & & & \\
\hline $\begin{array}{l}\text { 2.Feedback } \\
\text { contradiction }\end{array}$ & .729 & 1.000 & & & & & & & & & \\
\hline 3. Self-change & -.114 & -.221 & 1.000 & & & & & & & & \\
\hline 4.Self-concept clarity & -.509 & -.560 & .102 & 1.000 & & & & & & & \\
\hline 5.Dialectical self & .260 & .335 & -.129 & -.315 & 1.000 & & & & & & \\
\hline 6.Self-esteem & -.381 & -.464 & .225 & -.150 & -.327 & 1.000 & & & & & \\
\hline 7.Extraversion & -.173 & -.153 & .098 & .021 & -.159 & .373 & 1.000 & & & & \\
\hline 8.Openness & -.091 & -.115 & .171 & .164 & -.146 & .361 & .421 & 1.000 & & & \\
\hline 9.Consciensciousness & -.339 & -.319 & .078 & -.029 & -.269 & .426 & .289 & .324 & 1.000 & & \\
\hline
\end{tabular}




\begin{tabular}{|l|l|l|l|l|l|l|l|l|l|l|l|}
\hline 10.Neuroticism & .441 & .423 & -.105 & .153 & .333 & -.471 & -.377 & -.258 & -.478 & 1.000 & \\
\hline 11.Agreeableness & -.324 & -.308 & .179 & -.008 & -.142 & .355 & .354 & .272 & .377 & -.378 & 1.000 \\
\hline
\end{tabular}

\section{DISCUSSION}

Based on four independently recruited samples with a combined sample size of 1852 participants, the results of this study determined the factorial structure of the theoretically driven scale measuring self-contradiction and provided support for the reliability and the validity of this scale.

Specifically, The EFA and CFA conjointly determined a final version of self-contradiction scale with 16 items and 3 substantive dimensions. These results supported our hypothesized three-factor solution when method bias was controlled by specifying additional two method factors (for positively and negatively worded items, respectively). Method bias associated with item wording is a byproduct of adding negatively worded items to a scale, a simple device often used to "to disrupt nonsubstantive responding and to enable the detection and control of aberrant response behavior when it occurs" (Weijters et al., 2013), and could be parameterized by specifying method factors or allowing item uniqueness to inter-correlate. The presence of wording bias has been observed in a variety of scales with negatively worded items, such as Rosenberg's selfesteem scale (Carmines \& Zeller, 1979; Marsh, Scalas, \& Nagengast, 2010), life orientation test (Alessandri et al., 2010) and more recently, the self-sacrifice scale (Bélanger, Caouette, Sharvit, \& Dugas, 2014).

Results from Sample 3 and Sample 4 provided another aspect of psychometric evidence that supports the relative cross-temporal stability of the selfcontradiction scale across two time points. The crosstemporal correlations of Time 1 and Time 2 were smaller in Sample 4 than in Sample 3, probably because of the longer time span in Sample 4 during which participants' chance of encountering self-inconsistent feedbacks or experiences is greater.

Furthermore, this study also provided an initial evidence for the construct validity of self-contradiction. We examined the nomological network of the newly proposed construct by correlating the three dimensions of self-contradiction with a variety of psychological variables, including personality measures and other selfrelated constructs. Results indicated that Internal Contradiction and Feedback Contradiction correlated positively with dialectical self and negatively correlated with self-concept clarity, showing a good convergent validity. The scale also correlated with self-esteem and all five of the Big Five personality dimensions. Whereas internal and feedback contradictions negatively correlated with self-esteem, the self-change dimension showed a positive correlation with self-esteem. This result tallied with previous theories on self-consistency in which a low sense of self-worth would result when one is self-doubting or beset with self-uncertainty.

Besides, internal and feedback contradiction showed moderate positive correlations with neuroticism, and mild negative correlations with other four dimensions, suggesting that contradictions within both conceptual and phenomenal are associated with emotional instability and people with higher scores are less extraverted, less open to new experiences (fraught with more uncertainties), less conscientious and less agreeable than their more selfconsistent counterparts. The self-change dimension also showed significant correlations with the Big Five personalities yet with an opposite direction. The close relationship between one's level of self-contradiction and personalities is generally consistent with previous studies. In summary, the results of this study support our conceptualization of self-contradiction and the psychometric properties of the newly developed scale.

\section{CONCLUSION}

The present study developed and validated a new scale for the measurement of self-contradiction. The new scale showed good reliability and validity and is suitable for future research on self.

\section{ACKNOWLEDGMENTS}

This research was supported by the Fundamental Research Funds for the Central Universities (grant No. 2019WKYXQN18).

\section{REFERENCES}

[1] Alessandri, G., Vecchione, M., Fagnani, C., Bentler, P. M., Barbaranelli, C., Medda, E., . . . Caprara, G. V. (2010). Much more than model fitting? Evidence for the heritability of method effect associated with positively worded items of the Life Orientation Test revised. Structural Equation Modeling: A Multidisciplinary Journal, 17(4), 642-653. doi: 10.1080/10705511.2010.510064

[2] Bélanger, J. J., Caouette, J., Sharvit, K., \& Dugas, M. (2014). The psychology of martyrdom: Making the ultimate sacrifice in the name of a cause. Journal of Personality and Social Psychology, 107(3), 494515. doi: 10.1037/a0036855

[3] Campbell, J. D., Trapnell, P. D., Heine, S. J., Katz, I. M., Lavallee, L., \& Lehman, D. R. (1996). Selfconcept clarity: Measurement, personality correlates, and cultural boundaries. Journal of 
Personality and Social Psychology, 70(1), 141-156. doi: 10.1037/0022-3514.70.1.141

[4] Carmines, E. G., \& Zeller, R. A. (1979). Reliability and validity assessment. Beverly Hills, CA: Sage Publications, Inc.

[5] DiStefano, C., Zhu, M., \& Mîndrilă, D. (2009). Understanding and using factor scores: Considerations for the applied researcher. Practical Assessment, Research \& Evaluation, 14(20), 1-20.

[6] Fox, J. (2006). Structural equation modeling with the sem package in R. Structural Equation Modeling: A Multidisciplinary Journal, 13(3), 465-486. doi: 10.1207/s15328007sem1303 7

[7] Fox, J., Nie, Z., \& Byrnes, J. (2015). sem: Structural equation models (Version R package version 3.1-6). Retrieved from http://CRAN.Rproject.org/package $=$ sem

[8] Grice, J. W. (2001). Computing and evaluating factor scores. Psychological Methods, 6(4), 430-450. doi: $10.1037 / / 1082-989 x .6 .4 .430$

[9] John, O. P., Donahue, E. M., \& Kentle, R. L. (1991). The Big Five Inventory - Versions $4 a$ and 54. Berkeley, CA: University of California, Berkeley, Institute of Personality and Social Research.

[10] Lindwall, M., Barkoukis, V., Grano, C., Lucidi, F., Raudsepp, L., Liukkonen, J., \& ThøgersenNtoumani, C. (2012). Method effects: The problem with negatively versus positively keyed items. Journal of Personality Assessment, 94(2), 196-204. doi: 10.1080/00223891.2011.645936
[11] Marsh, H. W., Scalas, L. F., \& Nagengast, B. (2010). Longitudinal tests of competing factor structures for the Rosenberg Self-Esteem Scale: Traits, ephemeral artifacts, and stable response styles. Psychological Assessment, 22(2), 366-381. doi: 10.1037/a0019225.supp

[12] Meade, A. W., \& Craig, S. B. (2012). Identifying careless responses in survey data. Psychological Methods, 17(3), 437-455. doi: 10.1037/a0028085

[13] Podsakoff, P. M., MacKenzie, S. B., \& Podsakoff, N. P. (2012). Sources of method bias in social science research and recommendations on how to control it. Annual Review of Psychology, 63, 539-569. doi: 10.1146/annurev-psych-120710-100452

[14] R Core Team. (2015). R: A language and environment for statistical computing. Vienna, Austria: R Foundation for Statistical Computing. Retrieved from http:/www.R-project.org/

[15] Rosenberg, M. (1965). Society and the adolescent self-image. Princeton, NJ: Princeton University Press.

[16] Spencer-Rodgers, J., Boucher, H. C., Mori, S. C., Wang, L., \& Peng, K. (2009). The dialectical selfconcept: Contradiction, change, and holism in East Asian cultures. Personality and Social Psychology Bulletin, 35(1), 29-44. doi: $10.1177 / 0146167208325772$

[17] Weijters, B., Baumgartner, H., \& Schillewaert, N. (2013). Reversed item bias: An integrative model. Psychological Methods, 18(3), 320-334. doi: 10.1037/a0032121.supp 